\title{
Quadrature Formulas of Gaussian Type for Fast Summation of Trigonometric Series
}

\author{
Gradimir V. Milovanović*
}

\begin{abstract}
A summation/integration method for fast summing trigonometric series is presented. The basic idea in this method is to transform the series to an integral with respect to some weight function on $\mathbb{R}_{+}$and then to approximate such an integral by the appropriate quadrature formulas of Gaussian type. The construction of these quadrature rules, as well as the corresponding orthogonal polynomials on $\mathbb{R}_{+}$, are also considered. Finally, in order to illustrate the efficiency of the presented summation/integration method two numerical examples are included.
\end{abstract}

Keywords: Summation, trigonometric series, Gaussian quadrature rule, weight function, orthogonal polynomial, three-term recurrence relation, convergence, Laplace transform.

2010 Mathematics Subject Classification: 65B10, 41A55, 65D32.

\section{INTRODUCTION}

Let $\mathcal{P}$ be the space of all polynomials and $\mathcal{P}_{n}$ its subspace of polynomials of degree at most $n$. In a joint paper with Walter Gautschi [9], we developed the Gauss-Christoffel quadratures on $(0,+\infty)$,

$$
\int_{0}^{+\infty} f(t) w_{\nu}(t) \mathrm{d} t=\sum_{k=1}^{N} A_{\nu, k}^{(N)} f\left(\tau_{\nu, k}^{(N)}\right)+R_{N, \nu}(f) \quad(\nu=1,2),
$$

with respect to the Bose-Einstein and Fermi-Dirac weights, which are defined by

$$
w_{1}(t)=\varepsilon(t)=\frac{t}{\mathrm{e}^{t}-1} \quad \text { and } \quad w_{2}(t)=\varphi(t)=\frac{1}{\mathrm{e}^{t}+1},
$$

respectively. These $N$-point quadrature formulas are exact on the space of all algebraic polynomials of degree at most $2 N-1$, i.e., $R_{N, \nu}\left(\mathcal{P}_{2 N-1}\right)=0, \nu=1,2$.

The weight functions (1.2) and the corresponding quadratures (1.1) are widely used in solid state physics, e.g., the total energy of thermal vibration of a crystal lattice can be expressed in the form $\int_{0}^{+\infty} f(t) \varepsilon(t) \mathrm{d} t$, where $f(t)$ is related to the phonon density of states. Also, integrals with the second weight function $\varphi(t)$ are encountered in the dynamics of electrons in metals.

In the same paper [9], we showed that these quadrature formulas can be used for summation of slowly convergent series of the form

$$
T=\sum_{k=1}^{+\infty} a_{k} \quad \text { and } \quad S=\sum_{k=1}^{+\infty}(-1)^{k} a_{k}
$$

Received: 02 September 2019; Accepted: 17 October 2019; Published Online: 22 October 2019

*Corresponding author: Gradimir V. Milovanović; gvm@mi.sanu.ac.rs

DOI: $10.33205 / \mathrm{cma} .613948$ 
In general, the basic idea in such the so-called summation/integration procedures is to transform the sum to an integral with respect to some weight function $w(t)$ on $\mathbb{R}$ or $\mathbb{R}_{+}=$ $[0,+\infty)$, and then to approximate this integral by a finite quadrature sum, i.e.,

$$
\int_{\mathbb{R}} f(x) w(x) \mathrm{d} x \approx Q_{N}(f)=\sum_{\nu=1}^{N} A_{\nu}^{(N)} f\left(x_{\nu}^{(N)}\right),
$$

where the function $f$ is connected with $a_{k}$ in some way, and $x_{\nu}^{(N)}$ and $A_{\nu}^{(N)}, \nu=1, \ldots, N$, are nodes and weights of the quadrature rule $Q_{N}(f)$ (usually of Gaussian type), which is efficient for approximating a large class of functions with a relatively small number of quadrature nodes $N$.

As a transformation method of sums to integrals, we can use the Laplace transform as in [9] (see also $[5,8]$ ) or some methods of complex contour integration as in our papers $[12,13]$ (see also $[14,15,20,17])$. An account on summation/integration methods for the computation of slowly convergent power series and finite sums was given in [16].

In order to apply the quadrature rules (1.1) to the series $T$ and $S$ in (1.3), in the mentioned paper [9], we supposed that the general term of series is expressible in terms of the Laplace transform, or its derivative, of a known function. For example, let $a_{k}=F(k)$ and $F(s)=\mathcal{L}[f(t)]=\int_{0}^{+\infty} \mathrm{e}^{-s t} f(t) \mathrm{d} t$ for $\operatorname{Re} s \geq 1$. Then

$$
T=\sum_{k=1}^{+\infty} F(k)=\sum_{k=1}^{+\infty} \int_{0}^{+\infty} \mathrm{e}^{-k t} f(t) \mathrm{d} t=\int_{0}^{+\infty}\left(\sum_{k=1}^{+\infty} \mathrm{e}^{-k t}\right) f(t) \mathrm{d} t
$$

i.e.,

$$
T=\int_{0}^{+\infty} \frac{\mathrm{e}^{-t}}{1-\mathrm{e}^{-t}} f(t) \mathrm{d} t=\int_{0}^{+\infty} \frac{t}{\mathrm{e}^{t}-1} \frac{f(t)}{t} \mathrm{~d} t=\int_{0}^{+\infty} \varepsilon(t) \frac{f(t)}{t} \mathrm{~d} t .
$$

Similarly, for "alternating" series, we have

$$
S=\sum_{k=1}^{+\infty}(-1)^{k} F(k)=\int_{0}^{+\infty} \frac{1}{\mathrm{e}^{t}+1}(-f(t)) \mathrm{d} t
$$

where the Fermi-Dirac weight function $\varphi(t)$ on $(0,+\infty)$ is appeared on the right-hand side in (1.5).

In this way, the summation of the series $T$ and $S$ is transformed to the integration problems with respect to the weight functions $w_{1}(t)=\varepsilon(t)$ and $w_{2}(t)=\varphi(t)$, respectively. An application of quadrature formulas (1.1) for $\nu=1$ and $\nu=2$ to the integrals in (1.4) and (1.5), respectively, provides an acceptable procedure for summation of slowly convergent series $T$ and $S$.

In this paper, we consider the corresponding summation for the convergent trigonometric series

$$
C(x)=\sum_{k=1}^{+\infty} a_{k} \cos k \pi x \quad \text { and } \quad S(x)=\sum_{k=1}^{+\infty} a_{k} \sin k \pi x \quad(-1<x<1) .
$$

The corresponding series

$$
A(x)=\sum_{k=1}^{+\infty}(-1)^{k-1} a_{k} \cos k \pi x \quad \text { and } \quad B(x)=\sum_{k=1}^{+\infty}(-1)^{k-1} a_{k} \sin k \pi x,
$$

can be also considered, putting $x:=x-1$. Then $A(x)=-C(x-1)$ and $B(x)=-S(x-1)$. 
The series (1.6) can be treated in the complex form

$$
C(x)+\mathrm{i} S(x)=\sum_{k=1}^{+\infty} a_{k} \mathrm{e}^{\mathrm{i} k \pi x} .
$$

The paper is organized as follows. In Section 2, we present the transformation of (1.7) to the "weighted" integrals over $(0,+\infty)$. The construction of the corresponding quadrature formulas of Gaussian type for such integrals is given in Section 3. A simpler method for the sinus-series is presented in Section 4. Finally, in order to illustrate our methods, some numerical examples are given in Section 5.

\section{TRANSFORMATION OF (1.7) TO “WEIGHTED” INTEGRALS}

We consider the series (1.7) whose general term $a_{k}$ is expressible in terms of the Laplace transform of a known function, i.e., let $a_{k}=F(k)$, where

$$
F(s)=\mathcal{L}[f(t)]=\int_{0}^{+\infty} \mathrm{e}^{-s t} f(t) \mathrm{d} t, \quad \operatorname{Re} s \geq 1 .
$$

Then, we have

$$
C(x)+\mathrm{i} S(x)=\sum_{k=1}^{+\infty} \mathrm{e}^{\mathrm{i} k \pi x} \int_{0}^{+\infty} \mathrm{e}^{-k t} f(t) \mathrm{d} t=\pi \int_{0}^{+\infty}\left(\sum_{k=1}^{+\infty} \mathrm{e}^{-k \pi(t-\mathrm{i} x)}\right) f(\pi t) \mathrm{d} t,
$$

i.e.,

$$
C(x)+\mathrm{i} S(x)=\sum_{k=1}^{+\infty} a_{k} \mathrm{e}^{\mathrm{i} k \pi x}=\pi \int_{0}^{+\infty} \frac{\mathrm{e}^{\mathrm{i} \pi x}}{\mathrm{e}^{\pi t}-\mathrm{e}^{\mathrm{i} \pi x}} f(\pi t) \mathrm{d} t .
$$

The obtained integral on the right-hand side in (2.2) is weighted with respect to the one-parametar "complex weight function"

$$
w(t ; x)=\frac{\mathrm{e}^{\mathrm{i} \pi x}}{\mathrm{e}^{\pi t}-\mathrm{e}^{\mathrm{i} \pi x}}, \quad-1<x<1 .
$$

We note that

$$
w(t ; 0)=\frac{\varepsilon(\pi t)}{\pi t}, \quad w(t ; 1 / 2)=-\varphi(2 \pi t)+\frac{\mathrm{i}}{2 \cosh \pi t}, \quad \text { and } \quad w(t ; 1)=-\varphi(\pi t),
$$

where $\varepsilon(t)$ and $\varphi(t)$ are given by (1.2). As we can see, only for $x=0$ and $x= \pm 1$, the function $w(t ; x)$ is real. Also, $w(t ;-x)=\overline{w(t ; x)}$, so that it is enough to consider only the case when $0<x \leq 1$. The case $x=0$ is not interesting because it leads to a numerical series.

Lemma 2.1. The moments of the function (2.3) are given by

$$
\mu_{k}(x)=\int_{0}^{+\infty} t^{k} w(t ; x) \mathrm{d} t= \begin{cases}-\frac{1}{\pi} \log \left(1-\mathrm{e}^{\mathrm{i} \pi x}\right), & k=0, \\ \frac{k !}{\pi^{k+1}} \operatorname{Li}_{k+1}\left(\mathrm{e}^{\mathrm{i} \pi x}\right), & k \in \mathbb{N},\end{cases}
$$

where $\mathrm{Li}_{n}$ is the polylogarithm function defined by

$$
\operatorname{Li}_{n}(z)=\sum_{\nu=1}^{+\infty} \frac{z^{\nu}}{\nu^{n}}
$$


Proof. In order to calculate the moments (2.4), i.e., the integrals

$$
\mu_{k}(x)=\int_{0}^{+\infty} \frac{t^{k} \mathrm{e}^{\mathrm{i} \pi x}}{\mathrm{e}^{\pi t}-\mathrm{e}^{\mathrm{i} \pi x}} \mathrm{~d} t, \quad k \geq 0,
$$

we note that, for $a=\mathrm{e}^{\mathrm{i} \pi x}$, we have

$$
\frac{a}{\mathrm{e}^{\pi t}-a}=\frac{a \mathrm{e}^{-\pi t}}{1-a \mathrm{e}^{-\pi t}}=\sum_{\nu=1}^{+\infty} a^{\nu} \mathrm{e}^{-\nu \pi t} \quad\left(\left|a \mathrm{e}^{-\pi t}\right|=\mathrm{e}^{-\pi t}<1\right) .
$$

Then, we get

$$
\mu_{k}(x)=\sum_{\nu=1}^{+\infty} a^{\nu} \int_{0}^{+\infty} t^{k} \mathrm{e}^{-\nu \pi t} \mathrm{~d} t=\frac{k !}{\pi^{k+1}} \sum_{\nu=1}^{+\infty} \frac{a^{\nu}}{\nu^{k+1}},
$$

which is the desired result, having in mind (2.5).

Remark 2.1. An analytic extension of the function $\mathrm{Li}_{n}$ is given by

$$
\operatorname{Li}_{s}(z)=\frac{z}{\Gamma(s)} \int_{0}^{+\infty} \frac{t^{s-1}}{\mathrm{e}^{t}-z} \mathrm{~d} t \quad(|\arg (1-z)|<\pi) .
$$

The function $\mathrm{Li}_{n}$ is suitable for both symbolic and numerical calculation. It has a branch cut discontinuity in the complex $z$-plane running from 1 to $\infty$. This function is implemented in MATHEMATICA software as PolyLog $[n, z]$ and it can be evaluated to arbitrary numerical precision.

Seperating the real and imaginary parts in (2.3), i.e.,

$$
w(t ; x)=\frac{1}{2}\left\{\frac{\cos \pi x-\mathrm{e}^{-\pi t}}{\cosh \pi t-\cos \pi x}+\mathrm{i} \frac{\sin \pi x}{\cosh \pi t-\cos \pi x}\right\},
$$

and using (2.2), we obtain the following results:

Lemma 2.2. We have

$$
\begin{aligned}
C(x) & =\sum_{k=1}^{+\infty} a_{k} \cos k \pi x=\frac{\pi}{2} \int_{0}^{+\infty} \frac{\cos \pi x-\mathrm{e}^{-\pi t}}{\cosh \pi t-\cos \pi x} f(\pi t) \mathrm{d} t \\
S(x) & =\sum_{k=1}^{+\infty} a_{k} \sin k \pi x=\frac{\pi}{2} \int_{0}^{+\infty} \frac{\sin \pi x}{\cosh \pi t-\cos \pi x} f(\pi t) \mathrm{d} t
\end{aligned}
$$

where $a_{k}=F(k)$ and $f(t)=\mathcal{L}^{-1}[F(s)]$.

Remark 2.2. Similar formulas as in Lemma 2.2 are mentioned in [22, p. 725].

The real and imaginary parts of $2 w(t ; x)=w_{R}(t ; x)+\mathrm{i} w_{I}(t ; x)$ for different values of $x$ are presented in Figure 1.

As we can see, the imaginary part $t \mapsto w_{I}(t ; x)=\operatorname{Im}(2 w(t ; x))$ is a positive function on $\mathbb{R}_{+}$for each $0<x<1$, and all its moments are

$$
\mu_{k}^{I}(x)=\int_{0}^{+\infty} t^{k} \frac{\sin \pi x}{\cosh \pi t-\cos \pi x} \mathrm{~d} t= \begin{cases}1-x, & k=0 \\ \frac{2 k !}{\pi^{k+1}} \operatorname{Im}\left\{\operatorname{Li}_{k+1}\left(\mathrm{e}^{\mathrm{i} \pi x}\right)\right\}, & k \in \mathbb{N},\end{cases}
$$

so that the orthogonal polynomials with respect to the inner product

$$
(p, q)=\int_{0}^{+\infty} p(t) q(t) w_{I}(t ; x) \mathrm{d} t
$$



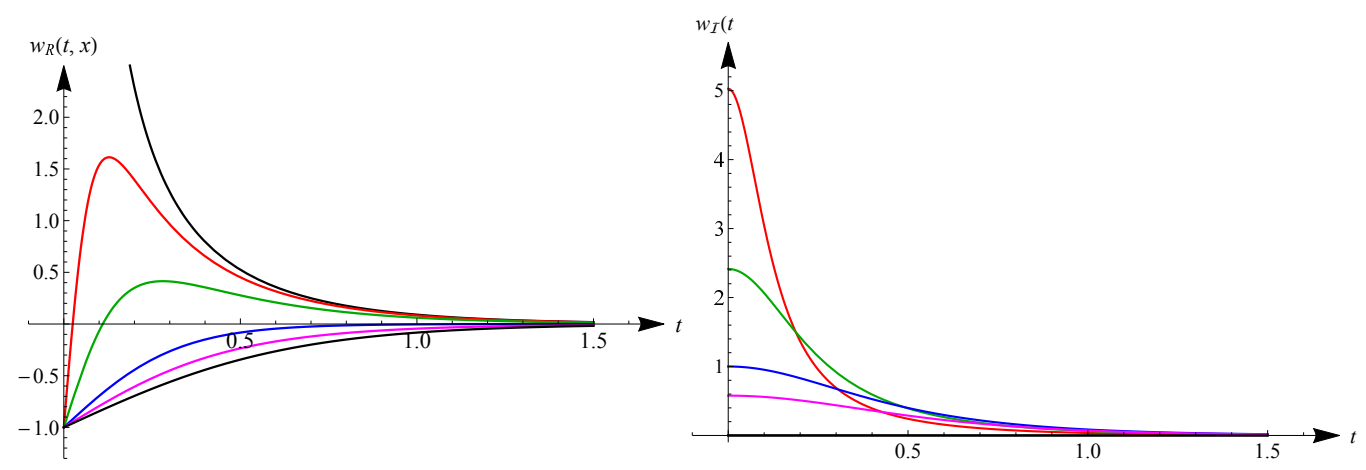

FIGURE 1. Real part (left) and imaginary part (right) of $t \mapsto 2 w(t ; x)$ on $[0,1.5]$ for $x=0$ (black line), $x=1 / 8$ (red line), $x=1 / 4$ (green line), $x=1 / 2$ (blue line), $x=2 / 3$ (magenta line), and $x=1$ (black line)

as well as the corresponding quadrature formulas of Gaussian type exist for each $n \in \mathbb{N}$.

However, the real part $t \mapsto w_{R}(t ; x)=\operatorname{Re}(2 w(t ; x))$ changes its sign at the point $t=$ $\pi^{-1} \log (1 / \cos \pi x) \in(0,+\infty)$, when $0<x<1 / 2$, while for $1 / 2 \leq x \leq 1$ this function is negative for each $t \in \mathbb{R}_{+}$. The moments of the function $w_{R}(t ; x)$ are

$$
\mu_{k}^{R}(x)=\int_{0}^{+\infty} t^{k} \frac{\cos \pi x-\mathrm{e}^{-\pi t}}{\cosh \pi t-\cos \pi x} \mathrm{~d} t= \begin{cases}-\frac{2}{\pi} \log \left(2 \sin \frac{\pi x}{2}\right), & k=0, \\ \frac{2 k !}{\pi^{k+1}} \operatorname{Re}\left\{\operatorname{Li}_{k+1}\left(\mathrm{e}^{\mathrm{i} \pi x}\right)\right\}, & k \in \mathbb{N} .\end{cases}
$$

Regarding these facts a system of orthogonal polynomials with respect to $t \mapsto w_{R}(t ; x)$ on $\mathbb{R}_{+}$exists for each $1 / 2 \leq x \leq 1$. However, for $0 \leq x<1 / 2$ the existence is not guaranteed.

\section{CONSTRUCTION OF POLYNOMIALS ORTHOGONAL WITH RESPECT TO THE WEIGHTS $t \mapsto w_{I}(t ; x)$ AND $t \mapsto w_{R}(t ; x)$ ON $\mathbb{R}_{+}$AND CORRESPONDING GAUSSIAN RULES}

As we mentioned in the previous section, the (monic) polynomials $p_{k}^{I}(t ; x), k=0,1, \ldots$, orthogonal with respect to the inner product (2.7) exist uniquely, as well as the corresponding quadrature formulas of Gaussian type

$$
\int_{0}^{+\infty} g(t) w_{I}(t ; x) \mathrm{d} t=\sum_{\nu=1}^{N} A_{\nu}^{I} g\left(\tau_{\nu}^{I}\right)+R_{N}(g ; x),
$$

where $\tau_{\nu}^{I}\left(\equiv \tau_{\nu}^{I}(N, x)\right)$ and $A_{\nu}^{I}\left(\equiv A_{\nu}^{I}(N, x)\right)$ are their nodes and weight coefficients. The corresponding remainder term $R_{N}(g ; x)$ vanishes for each $g \in \mathcal{P}_{2 n-1}$. Some error estimates of Gaussian rules for certain classes of functions can be found in [11, Sect. 5.1.5].

The monic polynomials $p_{k}^{I}(t ; x)$ satisfy the three-term recurrence relation

$$
p_{k+1}^{I}(t ; x)=\left(t-\alpha_{k}^{I}(x)\right) p_{k}^{I}(t ; x)-\beta_{k}^{I}(x) p_{k-1}^{I}(t ; x), \quad k=0,1, \ldots,
$$

with $p_{0}^{I}(t ; x)=1$ and $p_{-1}^{I}(t ; x)=0$. 
The nodes $\tau_{\nu}^{I}$ in the Gaussian quadrature rule (3.1) are eigenvalues of the symmetric tridiagonal Jacobi matrix (cf. [11, pp. 325-328])

$$
J_{N}\left(w_{I}(\cdot ; x)\right)=\left[\begin{array}{ccccc}
\alpha_{0}^{I}(x) & \sqrt{\beta_{1}^{I}(x)} & & & \mathbf{O} \\
\sqrt{\beta_{1}^{I}(x)} & \alpha_{1}^{I}(x) & \sqrt{\beta_{2}^{I}(x)} & & \\
& \sqrt{\beta_{2}^{I}(x)} & \alpha_{2}^{I}(x) & \ddots & \\
& & \ddots & \ddots & \sqrt{\beta_{N-1}^{I}(x)} \\
\mathbf{O} & & & \sqrt{\beta_{N-1}^{I}(x)} & \alpha_{N-1}^{I}(x)
\end{array}\right],
$$

and the weight coefficients $A_{\nu}^{I}$ are given by $A_{\nu}^{I}=\beta_{0}^{I}(x) v_{\nu, 1}^{2}, \nu=1, \ldots, N$, where $v_{\nu, 1}$ is the first component of the eigenvector $\mathbf{v}_{\nu}\left(=\left[\begin{array}{lll}v_{\nu, 1} & \ldots & v_{\nu, n}\end{array}\right]^{\mathrm{T}}\right)$ corresponding to the eigenvalue $\tau_{\nu}^{I}$ and normalized such that $\mathbf{v}_{\nu}^{\mathrm{T}} \mathbf{v}_{\nu}=1$. The most popular method for solving this eigenvalue problem is the Golub-Welsch procedure, obtained by a simplification of the QR algorithm [10].

Unfortunately, the coefficients in the three-term recurrence relation (3.2) are not known. They are known explicitly only for some narrow classes of orthogonal polynomials, including a famous class of the classical orthogonal polynomials (Jacobi, the generalized Laguerre, and Hermite polynomials). Orthogonal polynomials for which the recursion coefficients are not known are known as strongly non-classical polynomials. In the eighties of the last century, Walter Gautschi developed the so-called constructive theory of orthogonal polynomials on $\mathbb{R}$, including effective algorithms for numerically generating the recurrence coefficients for non-classical orthogonal polynomials, a detailed stability analysis of such algorithms as well as the corresponding software and several new applications of orthogonal polynomials (in particular see [4], [6], [7], as well as [18, 19, 20]).

On the other side, recent progress in symbolic computation and variable-precision arithmetic now makes it possible to generate the recurrence coefficients directly by using the original Chebyshev method of moments, but in a sufficiently high precision arithmetic. Such an approach allows us to overcome numerical instability in the map, in notation $\mathbf{K}_{n}: \mathbb{R}^{2 n} \rightarrow \mathbb{R}^{2 n}$, of the first $2 n$ moments to $2 n$ recursive coefficients,

$$
\boldsymbol{\mu}=\left(\mu_{0}^{I}(x), \mu_{1}^{I}(x), \ldots, \mu_{2 n-1}^{I}(x)\right) \mapsto \boldsymbol{\rho}=\left(\alpha_{0}^{I}(x) \ldots, \alpha_{n-1}^{I}(x), \beta_{0}^{I}(x), \ldots, \beta_{n-1}^{I}(x)\right),
$$

which is a major construction problem. Respectively symbolic/variable-precision software for orthogonal polynomials is now available: Gautschi's package SOPQ in MATLAB and our MATHEMATICA package OrthogonalPolynomials (see [3] and [21]), which is downloadable from the web site http://www.mi. sanu.ac.rs/ gvm/.

The package OrthogonalPolynomials, beside the numerical construction of the recurrence coefficients, enables also the construction in a symbolic form for a reasonable value of $n$. For example, executing the following commands

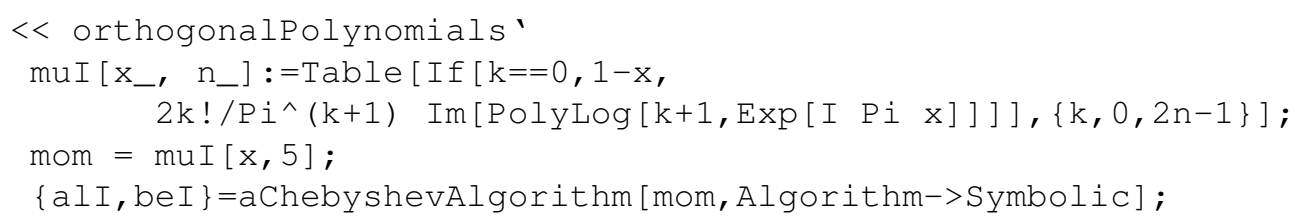

we obtain the first five coefficients $\alpha_{k}^{I}(x)$ and $\beta_{k}^{I}(x), k=0,1,2,3,4$, whose graphics are presented in Figure 2. 

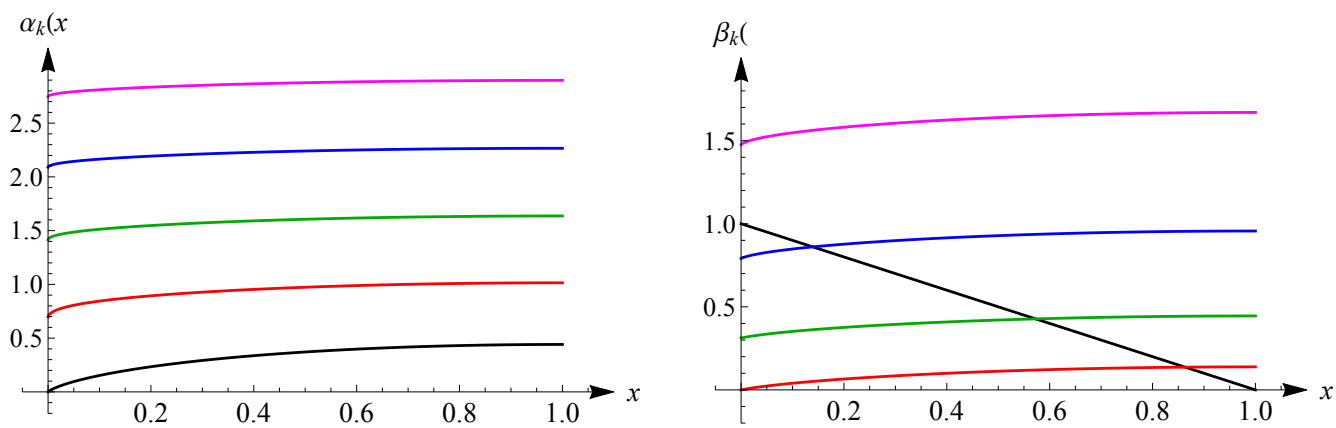

FIGURE 2. The coefficients $\alpha_{k}^{I}(x)$ (left) and $\beta_{k}^{I}(x)$ (right), for $k=0$ (black line), $k=1$ (red line), $k=2$ (green line), $k=3$ (blue line), and $k=4$ (magenta line)
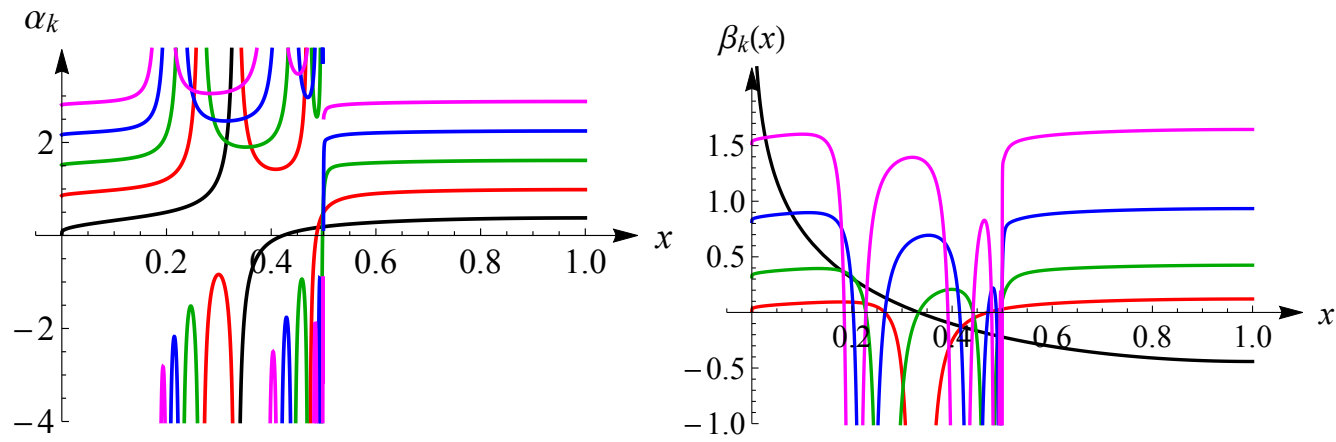

FIGURE 3. The coefficients $\alpha_{k}^{R}(x)$ (left) and $\beta_{k}^{R}(x)$ (right), for $k=0$ (black line), $k=1$ (red line), $k=2$ (green line), $k=3$ (blue line), and $k=4$ (magenta line)

Using these coefficients, we can calculate Gaussian parameters (nodes and weights) for each $N \leq n=5$ and each $x$. For larger values of $n$ and a given $x$, it is more convenient to use the option for numerical construction in the function aChebyshevAlgorithm, instead of symbolic construction. A numerical example is given in Section 5.

In the same way, we can obtain the graphics of the coefficients $\alpha_{k}^{R}(x)$ and $\beta_{k}^{R}(x), k=$ $0,1,2,3,4$, for the polynomials $p_{k}^{R}(t ; x), k=0,1, \ldots$, orthogonal with respect to the function $t \mapsto w_{R}(t ; x)$ on $\mathbb{R}_{+}$(see Figure 3 ). As we mention before, these polynomials exist uniquely for $1 / 2 \leq x \leq 1$, but for $0 \leq x<1 / 2$ their existence is not guaranteed.

\section{SOME CLASSES OF POLYNOMIALS ORTHOGONAL ON THE SEMIAXIS AND CORRESPONDING GAUSSIAN QUADRATURE RULES}

There are orthogonal polynomials related to Bernoulli numbers, discovered as early as Stieltjes [23] and later extended by Touchard [24] and Carlitz [1] (for details see Chihara [2, pp. 191-193]. Carlitz defined polynomials

$$
\Omega_{k}^{(\lambda)}(t)=\frac{(-1)^{k}(\lambda+1)_{k} k !}{2^{k}\left(\frac{1}{2}\right)_{k}} F_{k}^{\lambda}(1-\lambda+2 t),
$$


where, as usual $(\lambda)_{k}$ is the well known Pochhammer symbol (or the raised factorial, since $\left.(1)_{k}=k !\right)$, defined by

$$
(\lambda)_{k}=\frac{\Gamma(\lambda+k)}{\Gamma(\lambda)}=\lambda(\lambda+1) \cdots(\lambda+k-1),
$$

and $F_{k}^{\lambda}(t)={ }_{3} F_{2}\left[-k, k+1, \frac{1}{2}(1+\lambda+t) ; 1, \lambda+1 ; 1\right]$ is the so-called Pasternak polynomial. These polynomials are orthogonal (but not positive-definite) on a line in the complex plane $L=(c-\mathrm{i} \infty, c+\mathrm{i} \infty),-1<c<0$, with respect to the complex weight function $z \mapsto 1 /(\sin (\pi z) \sin \pi(z-\lambda))$. However, taking $(\lambda-1+\mathrm{i} t) / 2$ instead of $t$ (see [2, p. 192]), we get the positive-definite monic polynomials

$$
G_{k}^{(\lambda)}(t)=(-\mathrm{i})^{k} \Omega_{k}^{(\lambda)}\left(\frac{\lambda-1+\mathrm{i} t}{2}\right), \quad-1<\lambda<1,
$$

orthogonal with respect to the weight function

$$
t \mapsto w^{G}(t ; \lambda)=\frac{1}{\cosh \pi t+\cos \pi \lambda} \quad \text { on } \quad \mathbb{R}
$$

These polynomials satisfy the three-term recurrence relation

$$
G_{k+1}^{(\lambda)}(t)=t G_{k}^{(\lambda)}(t)-B_{k}(\lambda) G_{k-1}^{(\lambda)}(t), \quad G_{0}^{(\lambda)}(t)=1, G_{-1}^{(\lambda)}(t)=0
$$

where the recurrence coefficients are given by

$$
B_{0}(\lambda)=\int_{-\infty}^{+\infty} w^{G}(t ; \lambda) \mathrm{d} t=\frac{2 \lambda}{\sin \pi \lambda}, \quad B_{k}(\lambda)=\frac{k^{2}\left(k^{2}-\lambda^{2}\right)}{4 k^{2}-1}, \quad k=1,2, \ldots
$$

Remark 4.1. When $\lambda \rightarrow 0$ these polynomials $G_{k}^{(\lambda)}$ reduce to orthogonal polynomials with respect to the logistic weight $t \mapsto 1 /(\cosh \pi t+1)=2 \mathrm{e}^{-\pi t} /\left(1+\mathrm{e}^{-\pi t}\right)^{2}$ (see [16, p. 49]).

As we can see, there is a connection between the weights $w_{I}(t ; x)$ and $w^{G}(t ; \lambda)$ on $\mathbb{R}_{+}$. Namely,

$$
w_{I}(t ; x)=\sin (\pi x) w^{G}(t ; 1-x), \quad 0 \leq t<+\infty .
$$

Using this fact and some results from [11, pp. 102-103], we can get the recurrence coefficients in an explicit form for polynomials $M_{k}(t ; x)$ orthogonal with respect to the weight function

$$
t \mapsto w^{M}(t ; x)=\frac{\sin \pi x}{\sqrt{t}(\cosh \pi \sqrt{t}-\cos \pi x)} \quad \text { on } \quad \mathbb{R}_{+} \quad(0<x<1)
$$

Here, $\sin (\pi x)$ is a constant factor and it can be omitted.

Theorem 4.1. The polynomials $\left\{M_{k}(t ; x)\right\}_{k=0}^{+\infty}$ orthogonal with respect to the weight function $w^{M}(t ; x)$, given by (4.4), satisfy the three-term recurrence relation

$$
M_{k+1}(t ; x)=\left(t-\alpha_{k}^{M}(x)\right) M_{k}(t ; x)-\beta_{k}^{M}(x) M_{k-1}(t ; x), \quad k=0,1, \ldots,
$$


with $M_{0}(t ; x)=1$ and $M_{-1}(t ; x)=0$. The recurrence coefficients are

$$
\begin{cases}\alpha_{0}^{M}(x) & =\frac{1}{3} x(2-x), \\ \alpha_{k}^{M}(x) & =\frac{32(k+1) k^{3}-8 k^{2}(x-2) x-4 k(x-1)^{2}+(x-2) x}{(4 k-1)(4 k+3)}, \quad k \in \mathbb{N} ; \\ \beta_{0}^{M}(x) & =2(1-x), \\ \beta_{k}^{M}(x) & =\frac{4 k^{2}(2 k-1)^{2}\left(4 k^{2}-(1-x)^{2}\right)\left((2 k-1)^{2}-(1-x)^{2}\right)}{(4 k-3)(4 k-1)^{2}(4 k+1)}, \quad k \in \mathbb{N} .\end{cases}
$$

In terms of polynomials (4.1), these polynomials can be expressed in the form

$$
M_{k}(t ; x)=G_{2 k}^{(1-x)}(\sqrt{t}), \quad k=0,1,2, \ldots .
$$

Proof. According to (4.1), (4.2) and [11, Theorem 2.2.11], we conclude that $G_{2 k}^{(1-x)}(\sqrt{t})$ are monic polynomials orthogonal with respect to the weight function $t \mapsto w^{G}(\sqrt{t} ; 1-x) / \sqrt{t}$ on $\mathbb{R}_{+}$, so that (4.7) holds.

Now, using [11, Theorem 2.2.12] we obtain the coefficients in the three-term recurrence relation (4.8). As usual, we put (cf. [11, p. 97])

$$
\beta_{0}^{M}(x)=\int_{0}^{+\infty} w^{M}(t ; x) \mathrm{d} t=2(1-x) .
$$

Thus, we have $\alpha_{0}^{M}(x)=B_{1}(1-x)=x(2-x) / 3$, as well as

$$
\alpha_{k}^{M}(x)=B_{2 k}(1-x)+B_{2 k+1}(1-x) \quad \text { and } \quad \beta_{k}^{M}(x)=B_{2 k-1}(1-x) B_{2 k}(1-x),
$$

where the coefficients $B_{k}$ are given in (4.3). These formulas give the desired results.

Remark 4.2. A few first polynomials $M_{k}(t ; x)$ are

$$
\begin{aligned}
M_{0}(t ; x) & =1 \\
M_{1}(t ; x) & =t+\frac{1}{3} x(x-2), \\
M_{2}(t ; x) & =t^{2}+\frac{2}{7}\left(3 x^{2}-6 x-10\right) t+\frac{3}{35} x\left(x^{2}-4\right)(x-4), \\
M_{3}(t ; x) & =t^{3}+\frac{5}{11}\left(3 x^{2}-6 x-28\right) t^{2}+\frac{1}{11}\left(5 x^{4}-20 x^{3}-80 x^{2}+200 x+224\right) t \\
& +\frac{5}{231} x\left(x^{2}-4\right)\left(x^{2}-16\right)(x-6), \\
M_{4}(t ; x) & =t^{4}+\frac{28}{15}\left(x^{2}-2 x-18\right) t^{3}+\frac{14}{39}\left(3 x^{4}-12 x^{3}-100 x^{2}+224 x+648\right) t^{2} \\
& +\frac{4}{2145}\left(105 x^{6}-630 x^{5}-4830 x^{4}+23520 x^{3}+54824 x^{2}-158368 x-146112\right) t \\
& +\frac{7}{1287} x\left(x^{2}-4\right)\left(x^{2}-16\right)\left(x^{2}-36\right)(x-8),
\end{aligned}
$$

etc.

As an additional result, which will not be of interest in our summation of trigonometric series, we can prove the following statement: 
Theorem 4.2. The polynomials $\left\{N_{k}(t ; x)\right\}_{k=0}^{+\infty}$ orthogonal with respect to the weight function

$$
t \mapsto w^{N}(t ; x)=\frac{\sin \pi x \sqrt{t}}{\cosh \pi \sqrt{t}-\cos \pi x} \quad \text { on } \quad \mathbb{R}_{+} \quad(0<x<1)
$$

satisfy the three-term recurrence relation

$$
N_{k+1}(t ; x)=\left(t-\alpha_{k}^{N}(x)\right) N_{k}(t ; x)-\beta_{k}^{N}(x) N_{k-1}(t ; x), \quad k=0,1, \ldots,
$$

with $N_{0}(t ; x)=1$ and $N_{-1}(t ; x)=0$. The recurrence coefficients are

$$
\begin{aligned}
& \alpha_{0}^{N}(x)=\frac{1}{5}\left(-3 x^{2}+6 x+4\right), \\
& \alpha_{k}^{N}(x)=\frac{32(k+3) k^{3}-8 k^{2}\left(x^{2}-2 x-12\right)-12 k(x-3)(x+1)-3 x^{2}+6 x+4}{(4 k+1)(4 k+5)}, \\
& \beta_{k}^{N}(x)=\frac{4 k^{2}(2 k+1)^{2}\left(4 k^{2}-(1-x)^{2}\right)\left((2 k+1)^{2}-(1-x)^{2}\right)}{(4 k-1)(4 k+1)^{2}(4 k+3)}
\end{aligned}
$$

for each $k \in \mathbb{N}$. In terms of polynomials (4.1), the polynomials $N_{k}(t ; x)$ can be expressed in the form $N_{k}(t ; x)=G_{2 k+1}^{(1-x)}(\sqrt{t}) / \sqrt{t}$ for each $k \in \mathbb{N}_{0}$.

The coefficient $\beta_{0}^{N}(x)$ may be arbitrary, because it multiplies $N_{-1}(t ; x)=0$, but usually, it is appropriate to take

$$
\beta_{0}^{N}(x)=\int_{0}^{+\infty} w^{N}(t ; x) \mathrm{d} t=\frac{8}{\pi^{3}} \operatorname{Im}\left\{\operatorname{Li}_{3}\left(\mathrm{e}^{\mathrm{i} \pi x}\right)\right\} .
$$

In the sequel, we consider the Gaussian quadrature formula with respect to the weight function $w^{M}(t ; x)$ on $\mathbb{R}_{+}$,

$$
\int_{0}^{+\infty} g(t) w^{M}(t ; x) \mathrm{d} t=\sum_{\nu=1}^{N} A_{\nu}^{(N)}(x) g\left(\tau_{\nu}^{(N)}(x)\right)+R_{N}(g ; x),
$$

where $R_{N}(g ; x)$ is the corresponding remainder term $\left(g \in \mathcal{P}_{2 n-1}\right)$. As we mentioned in the previous section, the parameters of the quadrature formula (4.9), the nodes $\tau_{\nu}^{(N)}(x)$ and the weight coefficients $A_{\nu}^{(N)}(x)$, can be calculated very easy from the symmetric tridiagonal Jacobi matrix $J_{N}\left(w^{M}(\cdot ; x)\right)$ by the Golub-Welsch procedure. It is also implemented in the package OrthogonalPolynomials by the function aGaussianNodesWeights. Taking the recursion coefficients $\alpha_{k}^{M}(x)$ and $\beta_{k}^{M}(x), k=0,1, \ldots, n-1$, defined before in (4.6), we can calculate nodes and weights in (4.9) for a given $x$ and any $N \leq n$.

For calculating values of the series $S(x)$, presented in the form

$$
S(x)=\sum_{k=1}^{+\infty} a_{k} \sin k \pi x=\frac{\pi}{4} \int_{0}^{+\infty} \frac{\sin \pi x}{\sqrt{t}(\cosh \pi \sqrt{t}-\cos \pi x)} f(\pi \sqrt{t}) \mathrm{d} t,
$$

we use the quadrature rule (4.9). Thus, we approximate $S(x)$ by the quadrature sum $Q_{N}(f ; x)$, where

$$
Q_{N}(f ; x)=\frac{\pi}{4} \sum_{\nu=1}^{N} A_{\nu}^{(N)}(x) f\left(\xi_{\nu}^{(N)}(x)\right)
$$


and $\xi_{\nu}^{(N)}(x)=\pi \sqrt{\tau_{\nu}^{(N)}(x)}, \nu=1, \ldots, N$. The corresponding (relative) error is given by

$$
E_{N}(x)=\left|\frac{Q_{N}(f ; x)-S(x)}{S(x)}\right| \text {. }
$$

\section{NUMERICAL EXAMPLES}

Through two examples, we illustrate the efficiency of our methods. All computations were performed in Mathematica, Ver. 12, on MacBook Pro (15-inch, 2017), OS X 10.14.6.

Example 5.1. We consider the following series

$$
C(x)=\sum_{k=1}^{+\infty} \frac{k}{4 k^{2}-1} \cos k \pi x \quad \text { and } \quad S(x)=\sum_{k=1}^{+\infty} \frac{k}{4 k^{2}-1} \sin k \pi x .
$$

The sum of the first series is given by (cf. [22, p. 731])

$$
C(x)=-\frac{1}{4}-\frac{1}{4} \cos \frac{\pi x}{2} \log \left|\tan \frac{\pi x}{4}\right|,
$$

while for the sinus series, one can find that $S(x)=(\pi / 8) \cos (\pi x / 2)$.

Since

$$
f(t)=\mathcal{L}^{-1}\left[\frac{s}{4 s^{2}-1}\right]=\frac{1}{4} \cosh \frac{t}{2},
$$

using (2.2) and the corresponding Gaussian rules with respect to the weights $w_{R}(t ; x)$ and $w_{I}(t ; x)$, we have

$$
C(x)=\frac{\pi}{8} \sum_{\nu=1}^{N} A_{\nu}^{R} \cosh \left(\frac{\pi \tau_{\nu}^{R}}{2}\right)+R_{N}^{R}(x) \quad \text { and } \quad S(x)=\frac{\pi}{8} \sum_{\nu=1}^{N} A_{\nu}^{I} \cosh \left(\frac{\pi \tau_{\nu}^{I}}{2}\right)+R_{N}^{I}(x),
$$

respectively.

TABLE 1 . Relative errors $E_{N}^{I}(x)$ and $E_{N}^{R}(x)$, when $N=5,10,15,20$ and $x=0.1(0.1) 0.7$

\begin{tabular}{|c||l|l|l|l|l|l|l|}
\hline rel. err. & $x=0.1$ & $x=0.2$ & $x=0.3$ & $x=0.4$ & $x=0.5$ & $x=0.6$ & $x=0.7$ \\
\hline \hline$E_{5}^{I}(x)$ & $1.10(-5)$ & $2.03(-5)$ & $2.84(-5)$ & $3.54(-5)$ & $4.13(-5)$ & $4.62(-5)$ & $5.00(-5)$ \\
$E_{10}^{I}(x)$ & $2.40(-10)$ & $4.51(-10)$ & $6.39(-10)$ & $8.06(-10)$ & $1.10(-15)$ & $1.07(-9)$ & $1.16(-9)$ \\
$E_{15}^{I}(x)$ & $4.78(-15)$ & $9.07(-15)$ & $1.29(-14)$ & $1.64(-14)$ & $1.10(-15)$ & $2.19(-14)$ & $2.39(-14)$ \\
$E_{20}^{I}(x)$ & $9.14(-20)$ & $1.74(-19)$ & $2.50(-19)$ & $3.17(-19)$ & $1.88(-15)$ & $4.25(-19)$ & $4.64(-19)$ \\
\hline \hline$E_{5}^{R}(x)$ & $2.88(-5)$ & $6.78(-5)$ & $9.34(-5)$ & $2.67(-4)$ & $1.09(-8)$ & $2.57(-5)$ & $3.58(-5)$ \\
$E_{10}^{R}(x)$ & $6.40(-10)$ & $1.15(-9)$ & $9.49(-10)$ & $2.97(-9)$ & $5.16(-17)$ & $7.74(-10)$ & $8.14(-10)$ \\
$E_{15}^{R}(x)$ & $1.22(-14)$ & $2.25(-14)$ & $4.91(-14)$ & $8.44(-14)$ & $2.23(-25)$ & $1.16(-14)$ & $1.66(-14)$ \\
$E_{20}^{R}(x)$ & $5.86(-19)$ & $4.23(-19)$ & $8.73(-19)$ & $1.33(-18)$ & $9.12(-34)$ & $2.23(-19)$ & $3.21(-19)$ \\
\hline
\end{tabular}

The relative errors in these quadrature sums are given by

$$
E_{N}^{R}(x)=\left|\frac{R_{N}^{R}(x)}{C(x)}\right|=\left|\frac{1}{C(x)}\left(\frac{\pi}{8} \sum_{\nu=1}^{N} A_{\nu}^{R} \cosh \left(\frac{\pi \tau_{\nu}^{R}}{2}\right)-C(x)\right)\right|
$$

and

$$
E_{N}^{I}(x)=\left|\frac{R_{N}^{I}(x)}{S(x)}\right|=\left|\frac{1}{S(x)}\left(\frac{\pi}{8} \sum_{\nu=1}^{N} A_{\nu}^{I} \cosh \left(\frac{\pi \tau_{\nu}^{I}}{2}\right)-S(x)\right)\right|
$$


For getting recurrence parameters in the three-term recurrence relations for the polynomials $p_{k}^{R}(t ; x)$ and $p_{k}^{I}(t ; x), k=0,1, \ldots$, we apply the procedure described Section 3 . In order to save space the relative errors are given only at the points $x=j / 10, j=1, \ldots, 7$, for $N=5,10,15$ and 20 nodes in the Gaussian rules (see Table 1). Numbers in parentheses indicate the decimal exponents, e.g., $1.10(-5)$ means $1.10 \times 10^{-5}$. For $N=5$ we use the symbolic construction of recurrence coefficients, while for $N>5$ we use numerical construction with the WorkingPrecision $->50$, with repetitions for each $x$.

As we mention before, the existence of the orthogonal polynomials $p_{k}^{R}(t ; x)$, as well as the corresponding Gaussian formulas, are not guaranteed for $0 \leq x<1 / 2$. However, the obtained numerical results of $E_{N}^{R}(x), N=5(5) 20$, for selected values of $x \in$ $\{0.1,0.2,0.3,0.4\}$ show the existence of such quadrature rules, as well as a fast convergence.

Example 5.2. Now we consider the series

$$
S(x)=\sum_{k=1}^{+\infty} \frac{\sin k \pi x}{\left(1+k^{2}\right)^{1 / 2}}, \quad 0<x<1 .
$$

With $S_{n}(x)$ we denote the $n$-th partial sum, and by $e_{n}(x)$ its relative error, i.e.,

$$
e_{n}(x)=\left|\frac{S_{n}(x)-S(x)}{S(x)}\right| .
$$

The partial sums $S_{n}(x)$ are displayed in Figure 4 for $n=5,10$, and 50 . As we can observe their convergence is very slow.

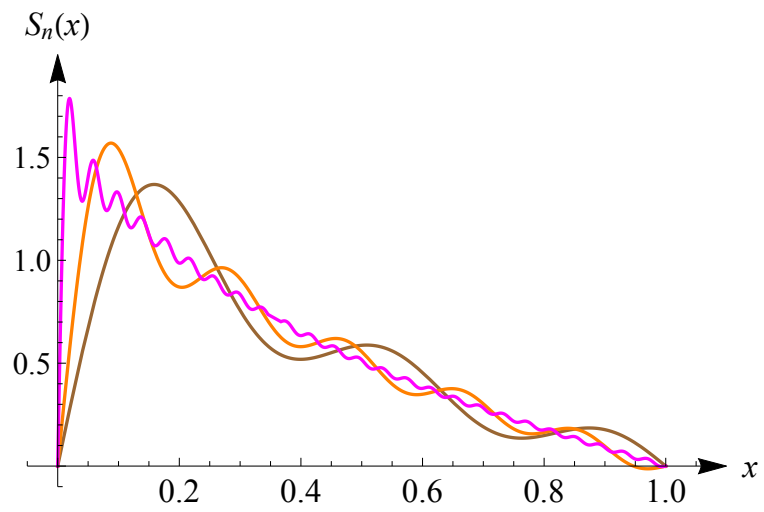

FIGURE 4. Partial sums $S_{n}(x)$ for $n=5$ (brown line), $n=10$ (orange line), and $n=50$ (magenta line)

Now we apply our method for summing trigonometric series. Using Lemma 2.2, we can identify $F(s)=\left(1+s^{2}\right)^{-1 / 2}$ and $f(t)=J_{0}(t)$, where $J_{0}$ is the Bessel function. Then, according to (4.10) and (4.11), we have

$$
\begin{aligned}
S(x)=\sum_{k=1}^{+\infty} \frac{\sin k \pi x}{\left(1+k^{2}\right)^{1 / 2}} & =\frac{\pi}{4} \int_{0}^{+\infty} \frac{\sin \pi x}{\sqrt{t}(\cosh \pi \sqrt{t}-\cos \pi x)} J_{0}(\pi \sqrt{t}) \mathrm{d} t \\
& \approx \frac{\pi}{4} \sum_{\nu=1}^{N} A_{\nu}^{(N)}(x) J_{0}\left(\xi_{\nu}^{(N)}(x)\right) .
\end{aligned}
$$


This quadrature process converges fast, because

$$
t \mapsto J_{0}(\pi \sqrt{t})=\sum_{m=0}^{+\infty} \frac{(-1)^{m}}{(m !)^{2}}\left(\frac{\pi}{2}\right)^{2 m} t^{m}
$$

is an entire function.

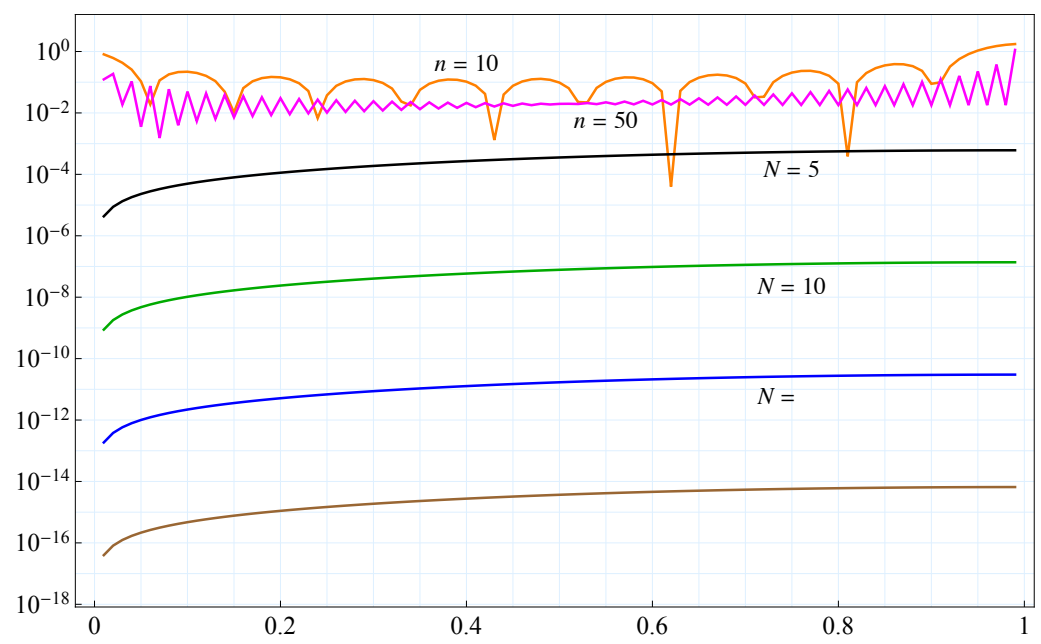

FIGURE 5. The relative errors $e_{n}(x)$ in the partial sums $S_{n}(x)$ for $n=10$ and $n=50$ and the relative errors $E_{N}(x)$ in the quadrature sums $Q_{N}(f ; x)$ for $N=5(5) 20$

The relative errors $E_{N}(x)$ of quadrature approximation $Q_{N}(f ; x)$ are given by (4.12), and presented in Figure 5 in log-scale for $N=5,10,15$, and 20 . Indeed, the convergence of $Q_{N}(f ; x)$ is fast. As we can see, the five-point Gaussian formula gives three to five exact decimal digits (depending of $x$ ) of the sum $S(x)$, and the quadrature formula with $n=20$ nodes gives an accuracy to more than 14 decimal digits.

TABLE 2. Relative errors $e_{n}(x)$ and $E_{N}(x)$, when $n=100$ and 500 and $N=5,10,20,50$, for some selected values of $x$ in $(0,1)$

\begin{tabular}{|c||c|c||c|c|c|c|}
\hline$x$ & $e_{100}(x)$ & $e_{500}(x)$ & $E_{5}(x)$ & $E_{10}(x)$ & $E_{20}(x)$ & $E_{50}(x)$ \\
\hline 0.1 & $2.49(-2)$ & $4.99(-3)$ & $4.96(-5)$ & $1.03(-8)$ & $4.72(-16)$ & $4.78(-38)$ \\
0.2 & $1.51(-2)$ & $3.03(-3)$ & $1.13(-4)$ & $2.39(-8)$ & $1.10(-15)$ & $1.13(-37)$ \\
0.3 & $1.12(-2)$ & $2.41(-3)$ & $1.88(-4)$ & $4.03(-8)$ & $1.88(-15)$ & $1.93(-37)$ \\
0.4 & $1.06(-2)$ & $2.12(-3)$ & $2.70(-4)$ & $5.87(-8)$ & $2.76(-15)$ & $2.84(-37)$ \\
0.5 & $9.87(-3)$ & $1.97(-3)$ & $3.54(-4)$ & $7.80(-8)$ & $3.68(-15)$ & $3.81(-37)$ \\
0.6 & $9.44(-3)$ & $1.89(-3)$ & $4.35(-4)$ & $9.66(-8)$ & $4.59(-15)$ & $4.76(-37)$ \\
0.7 & $9.18(-3)$ & $1.84(-3)$ & $5.06(-4)$ & $1.13(-7)$ & $5.39(-15)$ & $5.61(-37)$ \\
0.8 & $9.04(-3)$ & $1.81(-3)$ & $5.61(-4)$ & $1.26(-7)$ & $6.03(-15)$ & $6.28(-37)$ \\
0.9 & $8.96(-3)$ & $1.79(-3)$ & $5.96(-4)$ & $1.34(-7)$ & $6.44(-15)$ & $6.71(-37)$ \\
\hline
\end{tabular}

Also, the relative errors $e_{n}(x)$ in the partial sums $S_{n}(x)$ for $n=10$ and $n=50$ are shown in the same figure. Numerical values of the errors $e_{n}(x)$ in the partial sums $S_{n}(x)$ 
with $n=100$ and $n=500$ terms are given in the second and third column of Table 2 for equidistant values of $x(=0.1,0.2, \ldots, 0.9)$. We note that the number of exact digits in partial sums does not exceed three.

The numerical values of the corresponding relative errors $E_{N}(x)$ in the quadrature approximations $Q_{N}(f ; x)$, with $N=5,10,20,50$ nodes, at the same values of $x$ are given in the other columns of the same table. We note that the quadrature approximation $Q_{50}(f ; x)$ has about 37 exact decimal digits! As an exact value of $S(x)$ we use one obtained by the Gaussian quadrature formula with $N=100$ nodes.

\section{CONCLUSION}

In conclusion, we can say that the method presented in Section 3 is general for the both series $C(x)$ and $S(x)$, but for the sinus-series $S(x)$ the method presented in 4 is much simpler in applications, because in that case we have the recurrence relation (4.8), with recurrence coefficients in the explicit form (4.6).

\section{ACKNOWLEDGMENT}

The author was supported in part by the Serbian Academy of Sciences and Arts, Project No. $\Phi-96$.

\section{REFERENCES}

[1] L. CARLITZ: Bernoulli and Euler numbers and orthogonal polynomials. Duke Math. J. 26 (1959), 1-16.

[2] T. S. ChIHARA: An Introduction to Orthogonal Polynomials. Gordon and Breach, New York, 1978.

[3] A. S. Cvetković and G. V. Milovanović: The Mathematica package "OrthogonalPolynomials". Facta Univ. Ser. Math. Inform. 19 (2004), 17-36.

[4] W. GAUTSCHI: On generating orthogonal polynomials. SIAM J. Sci. Statist. Comput. 3 (1982), 289-317.

[5] W. GAUTSCHI: A class of slowly convergent series and their summation by Gaussian quadrature. Math. Comp. 57 (1991), 309-324.

[6] W. GAUTSCHI: Orthogonal polynomials: applications and computation. Acta Numerica (1996), 45-119.

[7] W. GAutSCHI: Orthogonal Polynomials: Computation and Approximation. Clarendon Press, Oxford, 2004.

[8] W. GAUTSCHI: The spiral of Theodorus, numerical analysis, and special functions. J. Comput. Appl. Math. 235 (2010), 1042-1052.

[9] W. GAUtSCHI and G. V. Milovanović: Gaussian quadrature involving Einstein and Fermi functions with an application to summation of series. Math. Comp. 44 (1985), 177-190.

[10] G. H. Golub and J. H. Welsch: Calculation of Gauss quadrature rule. Math. Comp. 23 (1969), 221-230.

[11] G. Mastroianni and G. V. Milovanović: Interpolation Processes - Basic Theory and Applications. Springer Monographs in Mathematics, Springer - Verlag, Berlin - Heidelberg, 2008.

[12] G. V. MiLovanović: Summation of series and Gaussian quadratures. In: Approximation and Computation (R.V.M. Zahar, ed.), pp. 459-475, ISNM Vol. 119, Birkhäuser Verlag, Basel-Boston-Berlin, 1994.

[13] G. V. Milovanović: Summation of series and Gaussian quadratures, II. Numer. Algorithms 10 (1995), 127-136.

[14] G. V. Milovanović: Summation processes and Gaussian quadratures. Sarajevo J. Math. 7 (20) (2011), 185-200.

[15] G. V. Milovanović: Quadrature processes and new applications. Bull. Cl. Sci. Math. Nat. Sci. Math. 38 (2013), $83-120$.

[16] G. V. Milovanović: Methods for computation of slowly convergent series and finite sums based on GaussChristoffel quadratures. Jaen J. Approx. 6 (2014), 37-68.

[17] G. V. Milovanović: On summation/integration methods for slowly convergent series. Stud. Univ. Babeş-Bolyai Math. 61 (2016), 359-375.

[18] G. V. Milovanović: Orthogonal polynomials on the real line, In: Walter Gautschi: Selected Works with Commentaries, Chapter 11, Volume 2 (C. Brezinski, A. Sameh, eds.), pp. 3-16, Birkhäuser, Basel, 2014.

[19] G. V. Milovanović: Computer algorithms and software packages, In: Walter Gautschi: Selected Works with Commentaries, Chapter 23, Volume 3 (C. Brezinski, A. Sameh, eds.), pp. 9-10, Birkhäuser, Basel, 2014.

[20] G. V. Milovanović: Construction and applications of Gaussian quadratures with nonclassical and exotic weight functions. Stud. Univ. Babeş-Bolyai Math. 60 (2015), 211-233. 
[21] G. V. Milovanović and A. S. CVetKović: Special classes of orthogonal polynomials and corresponding quadratures of Gaussian type. Math. Balkanica 26 (2012), 169-184.

[22] A. P. Prudnikov, Yu. A. BrychKov and O. I. MARIChev: Integrals and Series. Vol. 1. Elementary functions. Gordon \& Breach Science Publishers, New York, 1986.

[23] T. J. STIELTJES: Sur quelques intégrals definies et leur développement en fractions continues. Quart. J. Math. 24 (1890), 370-382 [Oeuvres. Vol. 2. Noordhoff, Groningen, 1918, pp. 378-394].

[24] J. TOUCHARD: Nombres exponentiels et nombres de Bernoulli. Canad. J. Math. 8 (1956), 305-320.

SERbian ACADEMy OF SCIENCES AND ARTS

11000 BELGRADE, SERBIA

AND

UNIVERSITY OF NIŠ

FACULTY OF SCIENCES AND MATHEMATICS

P.O. BOX 224, 18000 NIŠ, SERBIA

ORCID: 0000-0002-3255-8127

Email address: gvmemi.sanu.ac.rs 